\title{
La migración interna de los campesinos hacia las zonas urbanas en el Ecuador
}

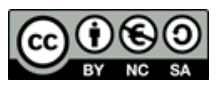

\section{The internal migration of peasants to urban areas in Ecuador}

\author{
Yolanda Tatiana Carrasco Ruano. ${ }^{1}$
}

Recibido: 15-06-2019 / Revisado: 17-06-2019 / Aceptado: 21-06-2019 / Publicado: 05-07-2019

\section{Resumen. $\quad$ DOI: $\underline{\text { https://doi.org/10.33262/concienciadigital.v2i3.932 }}$}

En la actualidad existen estudios de las migraciones internas en Ecuador que muestran que para entender las migraciones campo-ciudad es necesario comprender los factores que llevan a los campesinos a tomar una decisión riesgosa para él o ella y para el resto de su familia, factores entre las que se comprende la inestabilidad de su forma de vida habitual o tradicional, el cercamiento de las tierras comunales y en definidamente, la destrucción de sus medios para sobrevivir y sustentarse. Es decir, las personas de las zonas rurales abandonan los campos porque asumen que sus condiciones de vida mejorarán en las ciudades, pues, en éstas encontrarán al menos un mejor acceso a los servicios básicos de los cuales carecían en el campo. El 40,5 \% de los ecuatorianos radican en un lugar distinto al de su nacimiento, las personas que realizan un movimiento migratorio son básicamente adultos con bajo nivel de educación y por lo general dominan el español. El 87\% de los migrantes que no cuentan con ningún año de educación se mudan a ciudades con un nivel promedio de escolaridad, estas personas migran sin estar seguras de conseguir un empleo, simplemente se moviliza con la esperanza de acceder a un salario más alto que en la zona de origen. Sin embargo, una vez dentro de las urbes afrontan problemas como limitado acceso a viviendas por el elevado costo de alquiler, en consecuencia de aquello, los obliga a reubicarse en zonas marginales lo que provoca un incremento del perímetro de las ciudades.

Palabras claves: Migración interna, Campesinos, Zonas urbanas, Ecuador.

\footnotetext{
${ }^{1}$ Ciencia digital, Ambato, Ecuador, tcarrascor@yahoo.com
} 


\section{Abstract.}

At present there are studies of internal migrations in Ecuador that show that in order to understand the field-city migrations, it is necessary to understand the factors that lead the peasants to make a risky decision for him 88or her and for the rest of his family, factors between those who understand the instability of their habitual or traditional way of life, the enclosure of communal lands and in definite, the destruction of their means to survive and sustain themselves. That is, people from rural areas leave the camps because they assume that their living conditions will improve in the cities, because in these they will find at least better access to basic services that they lacked in the countryside. $40.5 \%$ of Ecuadorians reside in a different place than their birth, the people who make a migratory movement are basically adults with low level of education and usually dominate Spanish. $87 \%$ of migrants who do not have any years of education move to cities with an average level of schooling, these people migrate without being sure of getting a job, they simply move in the hope of accessing a higher salary than in the area of origin. However, once inside the cities they face problems such as limited access to housing due to the high cost of rent, as a consequence of that, it forces them to relocate to marginal areas, which causes an increase in the perimeter of the cities.

Keywords: Internal migration, Peasants, Urban areas, Ecuador.

\section{Introducción.}

\section{La migración interna de los campesinos hacia las zonas urbanas en el Ecuador.}

De acuerdo con los autores (Cisneros, C., Preston, D., Ibarra, H. (1985)., \&Cobo, (2004) Migraciones de campo a ciudad pequeña (o mediana). Es la primera secuencia de modalidad que al parecer es la dominante en la urbanización del país. Sus actores principales, sobre todo en la Sierra, 249 pertenecen probablemente a la clase social de los campesinos medios-o acomodados que aspiran a dejar su descendencia. Como en el caso de las migraciones temporales, que no implican desvinculación del migrante con su medio rural de origen. Migraciones de ciudad pequeña (o mediana) a ciudad grande o metrópoli. Es la secuencia que sigue a la anterior y sus actores son ex campesinos, hijos de campesinos, empleados, comerciantes urbanos, comerciantes profesionales, etc. Migraciones de campo a ciudad (mediana, grande o metrópoli), corresponden a las migraciones temporales e involucran, generalmente a campesinos jóvenes pauperizados que se ocupan tanto en los sectores formales (sobre todo tratándose de las migraciones hebdomadarias o diarias) o informales de las economías urbanas.

\section{Criterio personal.}

Los estudios sobre las migraciones internas en nuestro país pueden ayudarnos a entender y comprender cómo se formó nuestra nación. Así mismo cómo han ido evolucionando ciertas 
ciudades y, a su vez, sus habitantes. Todas estas posibilidades nos permiten afirmar que las migraciones internas son un tema relevante para el Desarrollo Rural, en cuanto nos permite aproximarnos a las dinámicas de los pobladores rurales quienes han sido los principales actores de las migraciones en Ecuador.

Una de las principales causas para que los jóvenes abandonen los campos se debe a los bajos salarios que se pagan en las actividades agropecuarias, que están muy distantes de los salarios que se pagan en las actividades de minería, la industria, el comercio y el transporte; y es por la escasa rentabilidad que obtienen los productores de alimentos.

Muchas viviendas en las zonas rurales están siendo abandonadas, debido a que los campesinos han emprendido viajes hacia las grandes urbes en busca de una nueva oportunidad de vida.

Algunos autores denominan a estos cambios como "aculturación", es aquella cuando hace referencia a todos esos cambios que se viven cuando se juntan o se ponen en contacto dos sociedades con tradiciones culturales diferentes.

La población rural sería la de mayor tendencia a migrar, hacia zonas metropolitanas o urbes y con mayor desarrollo. $\mathrm{Y}$ algunas zonas urbanas las mayores receptoras de migrantes. Algunas áreas atraen a la población ya sea por las posibilidades de encontrar un mejor trabajo o acceder a servicios como educación y salud. Y a su vez existen zonas consideradas deprimidas o malas dónde las personas no pueden trabajar o acceder a la tierra o no existen las instituciones públicas o privadas que puedan prestar los servicios básicos de la sociedad.

El 40,5\% de los ecuatorianos residen en un lugar distinto al de su nacimiento. De éstos, el $97 \%$ son ecuatorianos. Los migrantes internos provienen en su mayoría $(43,2 \%)$ de zonas rurales, el 19,8\% de áreas urbanas grandes, el 18,1\% de zonas urbanas medianas y el 18,8\% de áreas urbanas pequeñas. Ciudades como Quito y Guayaquil, con tasas de desempleo de $14,1 \%$ y $16,6 \%$ respectivamente, abarcan mayor cantidad de migrantes que Cuenca, cuya tasa de desempleo es $9,84 \%$. El $42 \%$ de los migrantes han tomado esta decisión motivados por determinantes familiares. El $31 \%$ migra por razones de trabajo. En porcentajes menos representativos se ubican razones como matrimonio (8\%), estudio (7\%), adquisición de inmuebles (4\%) salud (3\%), ingreso (3\%), clima (1\%). El movimiento migratorio si produce un efecto negativo en cuanto a las condiciones de vida fundamentalmente en las zonas urbanas. La investigación arroja como resultado que la condición de los migrantes en las zonas receptoras urbanas no es la más adecuada, es decir, las variables que demuestran la calidad de vida de los migrantes, como el acceso a servicios básicos y los ingresos, son desfavorables. 
Figura N 1: Causas de la migración.

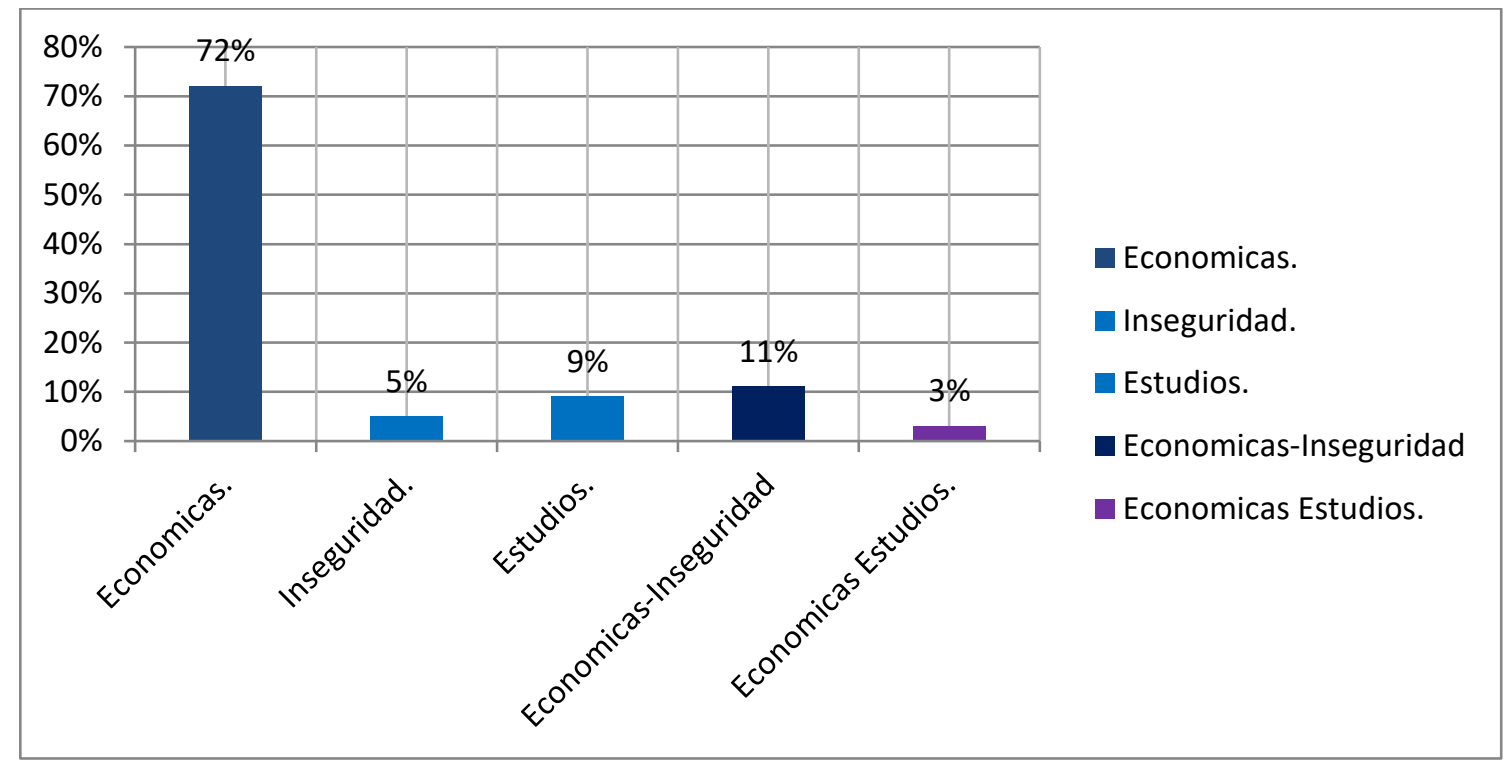

Fuente: https://es.slideshare.net/samanthajoako/la-migracion-interna-en-ecuador.

Aquí se puede visualizar fácilmente las causas por las que los campesinos deciden abandonar su lugar habitual de vida, para buscar nuevas formas de sustentar sus necesidades básicas que utilizan a lo largo de su existencia.

La principal causa para analizar es por la economía, ya que el campesino no cuenta con suficiente dinero para sustentar y satisfacer sus necesidades que necesita, a esto se le puede denominar como un problema o una dificultad que impide al desarrollo normal de una actividad. Otro aspecto a rescatar es la inseguridad que tiene la persona de la zona rural ya que no se siente seguro de habitar en una zona muy peligrosa, porque en los mayores de los casos el campesino suele tener sus viviendas construidas sobre las quebradas, eminencias topográficas y estas corren riesgo de ser destruidas en un sismo, por lo que su única opción es dejar el lugar y trasladarse a las grandes urbanizaciones.

En cuanto al estudio se puede decir que estas se dan en casos de jóvenes que residen en le campo y quieren sobresalir en el estudio hacen grandes viajes hacia las zonas urbanas para conseguir una buena educación y poder tener una capacidad para resolver sus problemas que tuvieron en las zonas rurales, por tanto, se debe tomar en cuenta que ellos buscan nuevos métodos para poder sobresalir en su vida. 
Figura N 2 Datos Estadísticos de la migración interna.

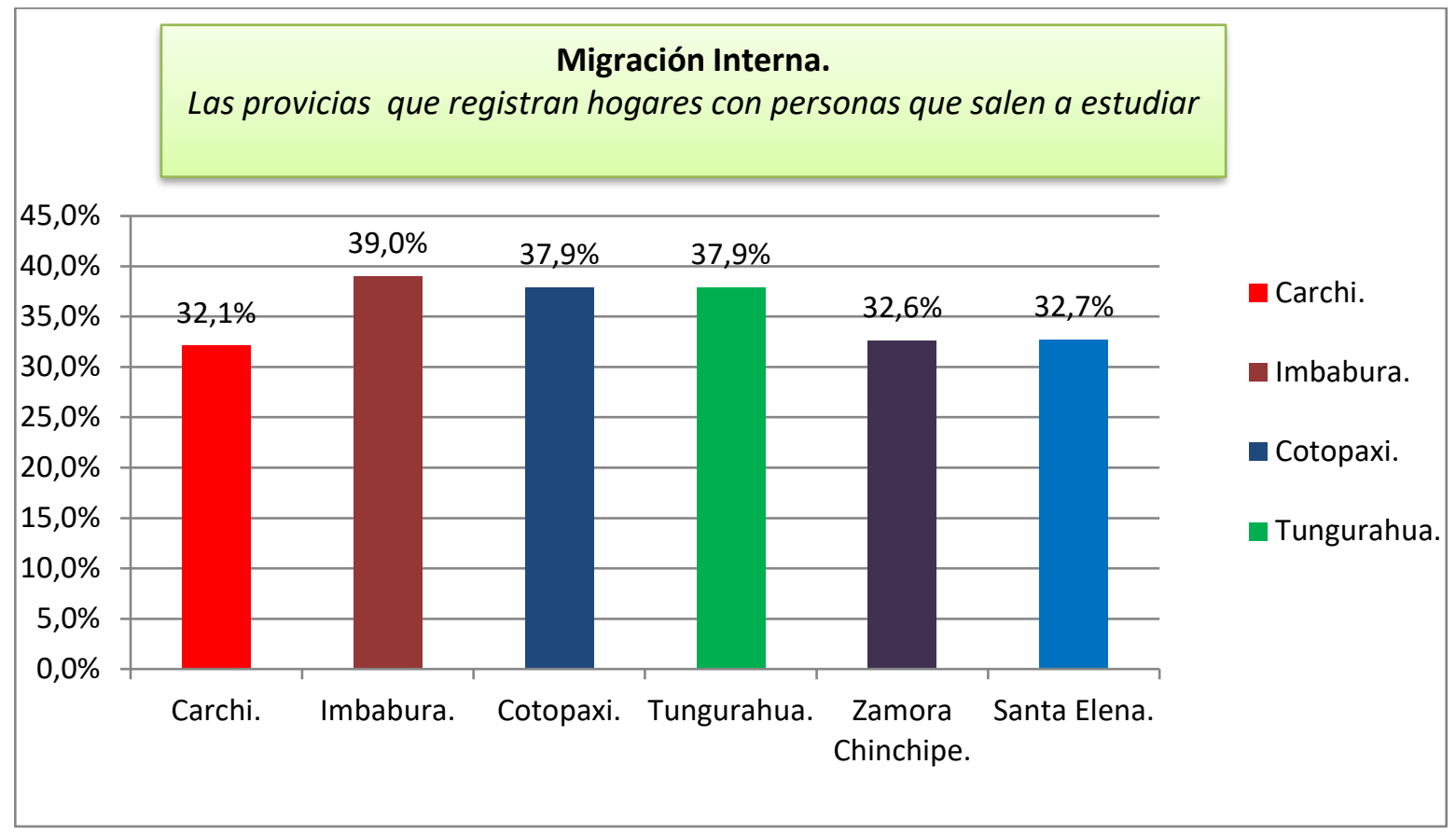

Fuente:https://www.elcomercio.com/actualidad/provincias-mayor-emigracionecuador.html

De acuerdo al análisis la mayoría de personas no solo salen en busca de trabajo, si no también salen con el propósito de Estudiar, así que no solo se puede tratar de la migración campesina.

En cuanto a la estadística analizada se puede resaltar que las provincias que mayormente se ven en la necesidad de salir en busca de trabajo o educación hacia las zonas urbanas son: Carchi, Imbabura, Cotopaxi, Tungurahua, Zamora Chinchipe y Santa Elena, estas provincias son las que más emiten campesinos hacia las grandes ciudades en busca de nuevas oportunidades de trabajo, vivienda, medicina, transporte, comunicación, economía, entre otras, con el fin de satisfacer sus propias necesidades, habrá casos que la persona tenga éxito en estos ámbitos que se mencionó anteriormente y en otros casos fracasaran, porque no todo suele suceder como uno piensa que va a resultar al tomar la difícil decisión de dejar el campo. 
Figura N 3: En el 27,3\% de los hogares tiene un integrante que sale para trabajar o estudiar.

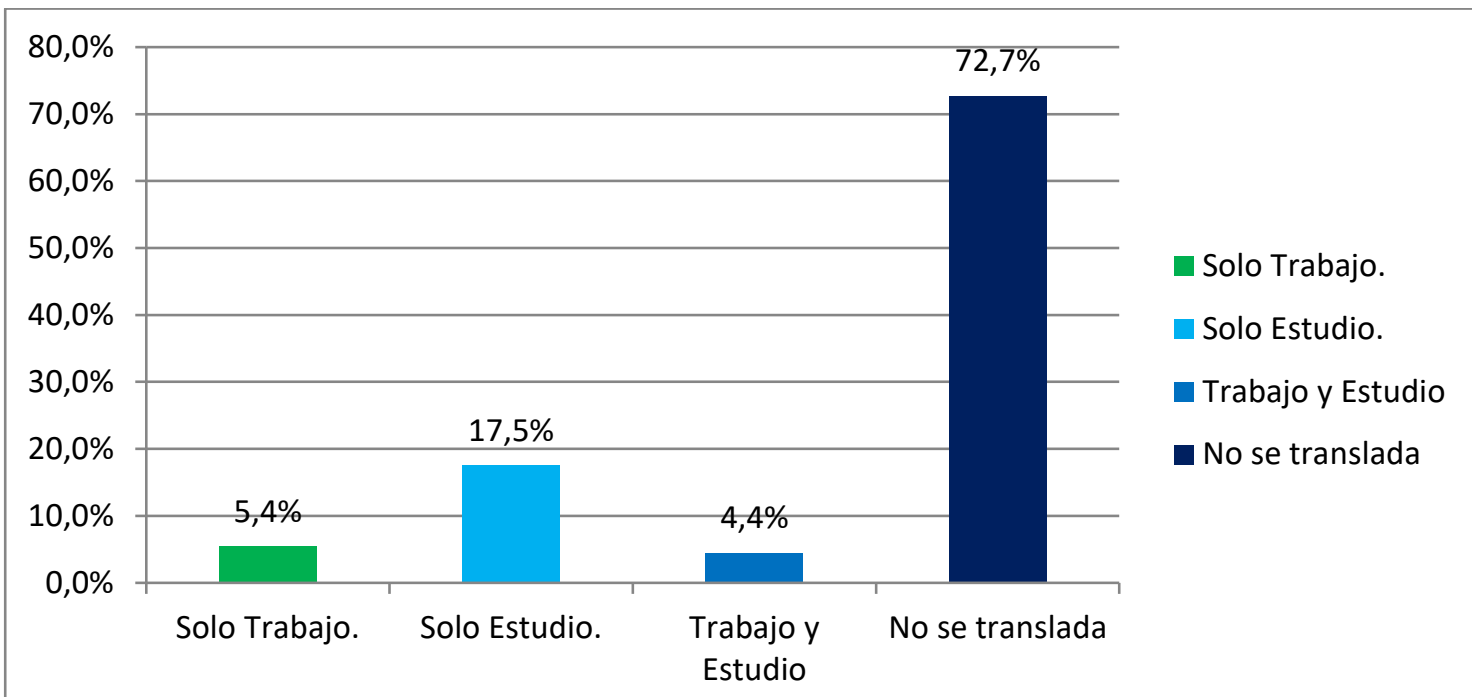

Fuente:https://www.elcomercio.com/actualidad/provincias-mayor-emigracionecuador.html

Respecto a este análisis estadístico se puede resaltar que 27,3 por ciento de hogares tienen un integrante que migran a otros lugares para trabajar, estudiar, en otros casos suelen salir toda la familia.

Con relación a esta estadística cabe señalar que del 100\%, el 72,7\% de las personas no se trasladan, por razones de que no quieren abandonar sus pertenencias, porque al momento de migrar ellos no cuentan con suficiente recursos económicos para sustentar en la ciudad y no correr riegos de inseguridad como el robo o la discriminación. Por otro lado tenemos con un total del 5,4\% de personas como lo había mencionado anteriormente solo buscan un trabajo digno para poder satisfacer sus necesidades tales como el agua, alimentación, medicina, vivienda, transporte como servicios principales para poder sobrevivir y mantener su calidad de vida. Consiguiente a los datos tenemos con un total del 17,5\% la personas que solo viajan por cuestiones de estudio que obligadamente deben abandonar su lugar de nacimiento en busca de una buena educación y superarse en la vida profesionalmente y por ultimo tenemos con un total del 4,4\%de la población viaja con el propósito de trabajar y al mismo tiempo a estudiar, que eso le revira como base de sustento de sus necesidades y superación profesional para identificarse como uno mismo ante toda la sociedad.

\section{Migración interna.}

De acuerdo a los autores (Sanz , N., Valenzuela, J. M. (2016)., Perruchoud.(2006)., Pérez\& Gardey.(2012). La migración interna es aquel movimiento de población hacia el territorio de 
otro Estado o dentro del mismo que abarca todo movimiento de personas sea cual fuese su tamaño, su composición o sus causas. Hace más de dos millones de años la migración no fue concebida como una solución desesperada, sino como la necesidad de dar respuesta a la curiosidad humana y de superar nuevos retos relacionados con nuestra capacidad de adaptación. Hoy, sin embargo, la migración se manifiesta como un fenómeno recurrente, cotidiano en los noticieros de todo el orbe, Además, la migración está referida a los penosos acontecimientos asociados a la violencia, al crimen organizado, a las guerras y a la imposibilidad de poner en práctica un ejercicio pleno de los derechos humanos.

\section{Criterio Personal.}

De acuerdo a la investigación es necesario resaltar que la migración interna es el movimiento o desplazamiento de personas dentro de un mismo lugar, ciudad o estado, por lo general esta suelen ser de las zonas rurales.

En este caso se relaciona a la migración de los campesinos que suelen abandonar su lugar habitual en busca de una mejora en la calidad de vida, en la cual emprenden un viaje hacia las zonas urbanas donde ellos pretenden mejorar sus condiciones de vida en las ciudades, pues en realidad, en éstas tienen la posibilidad de al menos contar con un mejor acceso a los servicios básicos de los cuales carecían en el campo, así como también aseguran que las oportunidades de empleo con una mayor remuneración son mejor que las del campo, pero siempre debe haber dificultades que limitan a que el campesino pueda sobresalir en la ciudad, porque una vez dentro de la ciudad ellos no cuentan con una vivienda y para ello los campesinos no cuentan con una alta tasa de economía para poder alquilar un apartamento por su alto valor de alquiler, u otro aspecto que suele darse con mayoría que es la discriminación, en consecuencia de aquello, y se ven obligados a reubicarse en las zonas marginales lo que provoca un incremento del perímetro de las ciudades.

Lo que significa un coste adicional para los gobiernos seccionales y por ende también para el estado. Estos costes se ven reflejado en las ampliaciones de los servicios, infraestructuras como carreteras, hospitales, centros educativos, etc.

Una de las ventajas que les ofrece las grandes ciudades a esta población migrante es la educación gratuita, ya que, en ocasiones, no cuentan con centros educativos en su comunidad, sector o lugar de origen, así como tampoco con servicios médicos adecuados. Por otra parte, existen la población que emigra proveniente de ciudades medianas donde ejercían algún oficio o alcanzaron cierto nivel educativo, incluso algunos son profesionales, aunque estos grupos son minoritarios.

Cabe resaltar que la migración es un fenómeno social global causado por la búsqueda de mejores oportunidades económicas y laborales. La movilidad de lugares rurales a urbanos representa cambios a nivel individual, familiar y comunitario, los cuales impactan en la 
conducta, hábitos, valores, actitudes, estilos y perspectivas de vida habitual que salían tener en el campo. Estas circunstancias son factores de riesgo que promueven problemas de salud pública, entre ellos el uso de drogas y los trastornos mentales, los cuales requieren de servicios de salud.

Además es un problema presente a lo largo de la historia de los seres humanos, por lo que se debe tratar dos puntos importantes como son: la migración temporal la cual consiste en la salida de los jóvenes o cualquier miembro de la familia fuera de la comunidad por un período no mayor a un año calendario etc. Y por otra parte la migración permanente que se entiende por la salida definitiva de algunos miembros de una familia o de la familia entera fuera de la comunidad. Este tipo de migración es por lo general hacia zonas urbanas donde fijan su asentamiento y la mayoría de ellos realiza su primera incursión en el sector de la construcción.

Estos sucesos empañan una parte fundamental de lo que todos somos como seres migrantes por naturaleza y por cultura.

\section{Campesino.}

De acuerdo a los autores(Aguirre, F.(1998).,Wolf. E.(1966).,Pérez, J., \& Gardey, A. (2017). Se emplea para denominar a aquel o aquello vinculado al campo: las tierras que se encuentran fuera de una ciudad y que, por lo general, son laborables. El concepto suele aludir al individuo que reside y desarrolla actividades en un campo. Los campesinos son quienes integran una comunidad rural. Pueden ser propietarios de la tierra que trabajan u obreros contratados por el dueño del terreno para realizar tareas de cultivo, ganadería, etc. El término campesino suele tener ciertas connotaciones negativas en el habla cotidiana, no sólo de las personas criadas en la ciudad sino incluso de algunas que nacieron en el campo. Lamentablemente, los medios de comunicación alimentan la idea de que los campesinos son seres inferiores, ya sea por su formación académica o por sus intereses, y nos llevan a creer que se trata de esclavos que se encuentran al servicio de los habitantes de las grandes ciudades.

\section{Criterio personal.}

Entendido la información cabe recalcar que campesino se refiere a una persona perteneciente o que vive en el campo, una de las definiciones más importantes que se debe recalcar es: Los campesinos son cultivadores rurales cuyos excedentes se transfieren al grupo dominante de los gobernantes, quienes emplean los excedentes para asegurar su propio nivel de vida y para distribuir el restante a grupos de la sociedad no rurales que requieren de alimentos a cambio de sus bienes.

En otros términos son personas que se encuentran fuera de la urbanización. En este caso se le va a regir con la migración dentro de Ecuador, ya que el campesino tiene la necesidad de querer realizar un viaje en busca de una mejora en la calidad de vida en zonas urbanas, 
debido a que el campesino piensa que en la zona habitual (campo) no cuenta con servicios necesarios, él o ella pretende buscar medios para poder sustentar sus necesidades básicas como la vivienda, alimentación, medicina, entre otras. Para ello se debe tomar en cuenta que no todo va a resultar como él o ella piensa, ya que existirá limitantes que le dificultan a sobresalir y triunfar en lo que se planifico antes de abandonar el campo, debido a su poco conocimiento el individuo no suele adaptarse con rapidez a las zonas urbanas, debido al caso surge una consecuencia que es la marginación por parte de los habitantes que residen en dicho lugar, la persona se sentirá inseguro o insegura y no podrá realizar sus actividades diarios con precisión.

Por un lado el campesino forma parte de la clase diligente, explotada y marginada en el proceso productivo, ajena a la posesión de propiedad y doblemente explotado por las redes comerciales y marginados por que su condición lo aleja de las posibilidades de gozar de los servicios públicos y sociales, que aun con dificultades pueden gozar los trabajadores de las ciudades.

La media parte de personas que sufren de necesidad alimenticia son pequeñas agricultoras y agricultores privados de un acceso adecuado a los recursos productivos, como tierra, agua, créditos y semillas. De estos, dos tercios vive en tierras marginales y bajo condiciones medioambientales difíciles: en colinas o zonas amenazadas por sequías u otras catástrofes naturales como inundaciones y corrimientos de tierras.

Cabe destacar que los campesinos son cultivadores rurales cuyos excedentes se transfieren al grupo dominante de los gobernantes, quienes emplean los excedentes para asegurar su propio nivel de vida y para distribuir el restante a grupos de la sociedad no rurales que requieren de alimentos a cambio de sus bienes.

Por otra parte está sujeta en base a su sistema de relación con el mundo interior. Un agricultor rural es campesino cuando mantiene una relación de dependencia respecto al resto de la sociedad, al que por supuesto permanece integrado, en términos políticos, económicos y culturales, un campesino produce básicamente para su conservación y a la de los suyos, para mantener asegurada su producción y consumo futuro.

\section{Zonas Urbanas.}

De acuerdo con los autores (Mari., A. (2007)., Peréz \& Merino, 2012) campesino Hace referencia a aquello perteneciente o relativo a la ciudad. Una ciudad es un área con una alta densidad de población y cuyos habitantes, por lo general, no se dedican a las actividades agrícolas.

Las ciudades presentan características como el predominio de las viviendas verticales y colectivas (los edificios), el poco terreno destinado a los espacios verdes y la buena infraestructura en materia de transportes y comunicaciones. Es posible hablar de zona urbana 
para nombrar a aquella región que cuenta con más de 2.000 habitantes, en su mayoría dedicados al sector secundario (industrial) o terciario (servicios) de la economía. Ante el creciente desarrollo urbano, la delimitación de una zona urbana contempla diferentes factores que varían de acuerdo al país.

\section{Criterio Personal.}

Haciendo referencia a zona rural se puede destacar que son lugares relativos a las grandes ciudades, con un alto índice de habitantes, las personas que residen en estos lugares no se dedican a las actividades o producción agrícola, en estas zonas las características que se pueden evidenciar fácilmente son las estructuras de forma vertical que son conocidas como edificios, estas zona de urbanización cuentan con medios de transporte, comunicación, infraestructuras educativas, hospitales, entre otras, que estas están dirigidas para el servicio público, A diferencia de las zonas rurales estos lugares siempre están en constante crecimiento incluso estas implementan día a día nuevas modalidades como por ejemplo las famosas ciudades inteligentes, para considerar una zona urbana esta debe estar habitada por más de dos mil personas.

En el marco del análisis de los problemas relacionados con los asentamientos urbanos ha tomado particular importancia el fenómeno de la urbanización. Esto se ha asimilado con el hecho de que continuamente incrementa el número de habitantes, de una nación o región, que reside en áreas en que se dispone de mayores y mejores servicios de enseñanza, sanidad e higiene, esparcimiento, agua, y electricidad, etc.

Este fenómeno ha cobrado especial interés a partir de la revolución industrial. El desarrollo económico, impulsado por los adelantos técnicos, se ha caracterizado por la concentración de las actividades industriales y de las ventajas del crecimiento en unas áreas denominadas urbanas.

El espacio urbano creció por la emigración mayormente procedente del rural, y su estilo de vida se extendió no solo por Ecuador sino también por todo el mundo. Además en estos lugares se pueden encontrar las grandes industrias producen productos para el consumo humano, a veces existen mayores posibilidades de encontrar empleo, numerosos servicios sociales y mayores ofertas culturales y de ocio. En el Ecuador existen 12 Grandes urbes como son Quito, Cuenca, Ambato, Loja, Santo Domingo, Guayaquil, Esmeraldas, Quevedo, Duran, Manta, Portoviejo y Machala, la mayor parte de estas ciudades reciben a los migrantes campesinos, quienes optan por adaptarse al lugar y esperar el futuro que ellos tendrán para sobrevivir junto a su familia, y para ello estas personas deben ser perseverantes y dar lo mejor de uno mismo.

La mayoría de la población se concentra en ciudades, como consecuencia del desarrollo industrial. En el siglo XX, su número aumentó considerablemente, y muchas llegaron a 
albergar a millones de habitantes. El espacio urbano creció por la migración procedente de zonas rurales, y su estilo de vida se extendió por todo el mundo.

Comúnmente las industrias ocupan espacios alrededor del $40 \%$ de la población. En el sector terciario trabaja alrededor del 50\%, Existen mayores posibilidades de encontrar empleo, numerosos servicios sociales y mayores ofertas culturales y de ocio. Poseen una periferia cada vez más urbanizada, lo que ha facilitado la creación de amplias vías de comunicación y el desarrollo del transporte público, entre otros para el beneficio de cada persona habitante del lugar. Pero en consecuencia se generan grandes cantidades de desechos y residuos gaseosos causados por automóviles, industrias, calefacciones. Además, se consumen grandes cantidades de agua, alimentos y energía, afectando a la disminución de los recursos naturales, obteniendo como resultado la degradación y la polución del medio ambiente, debido a que se liberan grandes cantidades de desechos tóxicos a la atmosfera y al ecosistema donde habitamos.

\section{Ecuador.}

De acuerdo a los autores (Cunti, 2007\& Ana, 2018) La misión geodésica francesa se realizó en nuestro país en lo que se conocía la Real Audiencia de Quito en el siglo XVII. Su principal objetivo era comprobar la forma de la tierra. Se utilizaron como base los estudios realizados por geógrafos reconocidos como Newton, Cassini, Picard, Colbet, Merchaut, Delambre, Snelius, Clairaut. En 173, luego de que el rey de España, Felipe V, otorga el permiso, pues el territorio era una colonia española en América, la misión llego a estas tierras y sus estudios concluyeron en 1745.

Como resultado de este trabajo se comenzó a identificar a los alrededores de Quito como las tierras del Ecuador, en referencia a la línea que divide el planeta en dos hemisferios, La MisiónGeodésica Francesa tuvo un aporte importante de carácter científico y además permitió que el mundo conozca el nombre de Ecuador y se puedan compartir aspectos de carácter social, cultural y antropológico.

\section{Criterio Personal.}

De acuerdo a la información obtenida cabe señalar que ecuador es uno de los países que conforma en Sudamérica. En la actualidad está conformada por veinte y cuatro provincias y su principal atractivo es la Mitad del Mundo y la mayoría de nuestros compatriotas dominan el español y un pequeño porcentaje hablan otros idiomas como el Kichwa, paicoca, awapit, entre otras. Cuenta con la principal cadena montañosa conocida como la cordillera de los andes. Comprende hechos importantes en cuanto a la historia a lo largo de su tiempo recorrido, Su nombre proviene después de haber concluido acerca de la teoría de la línea ecuatorial o equinoccial entre los años de 1736 y 1744, hecha por la misión geodésica 
francesa dirigida por Charlie Merie de la Condamine, quien viajo a ecuador para resolver sobre si el globo terráqueo tenia achatamientos en los polos y los extremos.

Ecuador, una vez independizado de España, se presentó al mundo como un país no sólo dividido en regiones -litoral, sierra y oriente- sino también con propuestas sociales, políticas e ideológicas diferentes. De hecho, la región costeña, además de ofrecer una fuerte tendencia agrícola, expresándose a través de las grandes plantaciones de cacao y de caña de azúcar, comenzó igualmente un moderado proceso de industrialización.

Cosa que no sucedió en la sierra por cuanto el latifundio y la población indígena que estaban en control de la hacienda, marcaron profundamente el desarrollo de las sociedades que en esta región ecuatoriana se fueron conformando.

El país cuenta con una gran variedad de biodiversidad que no se puede encontrar en cualquier lugar, la mayor producción para el ingreso de devisas es el petróleo, por otra parte también tenemos al turismo como una fuente dinamizadora para generar ingresos económicos, además se exportan productos como: el camarón, banano, cacao, rosas que están destinadas al extranjero y producen un ingreso de capital para el desarrollo del país.

Además comprende la diversidad de etnias, cultura, costumbres que existen en nuestro país, que hacen que nos represente como un país único en el mundo. Por consiguiente es necesario recalcar que en las zonas rurales las personas agricultores viven y producen la materia prima para el sustento de su alimentación, que luego se comercializan a nivel nacional y algunos productos a nivel internacional.

En cuanto a la historia del Ecuador cabe recalcar que es un conjunto de sucesos en el tiempo, en el cual los territorios que actualmente pertenecen a la República del Ecuador cambian en su aspecto físico y forma de gobierno. La historia de la República puede dividirse en cuatro etapas: Etapa Prehispánica, Etapa Hispánica (Conquista, colonización y Colonia), Independencia y República. El comienzo de la Historia de Ecuador se da a partir de las organizaciones prehispánicas que terminan con la Invasión Incaica, luego de esto surge la Conquista Española para luego con las fundaciones de San Francisco de Quito, San Gregorio de Portoviejo y Santiago de Guayaquil empezar una nueva era político-administrativa española que duraría hasta la época de la independencias cuando surge la nación colombiana de Simón Bolívar y luego al dividirse formaría lo que hoy se llama República del Ecuador.

\section{Conclusiones}

- Se llega a la conclusión de que la migración campesina hacia las zonas urbanas tienen un ritmo continuo, ya que los campesinos viajan a las grandes ciudades en busca de una mejora de vida y se seguirán dando mientras no se creen medidas para mejorar 
las estrategias de producción que sustenten al desarrollo y el bienestar de la comunidad.

- Zonas urbanas comprenden las grandes ciudades habitualmente habitadas por una gran cantidad de personas, estas grandes urbes están conformadas por inmensos edificios y de comunicación, la cual brindan la hospitalidad necesaria, pero para la persona campesina a veces es difícil acceder a este tipo de vivienda por su alto valor de alquiler.

- En el ecuador existen altos números de migración campesina, que abandonan el campo para buscar nuevos métodos de empleo y generar divisas que ayuden a sustentar su modo habitual de vida y al mismo tiempo satisfacer su necesidad que él tiene que asumir como persona, dentro de esto existe un punto negativo que es la marginación por parte de los habitantes de las urbes, dando así como consecuencia el esparcimiento y la extensión de territorio, haciendo que, los gobiernos deben crear nuevas infraestructura y nuevos gastos económicos para el estado.

- Ecuador está conformada por veinte y cuatro provincias, que brindan gran diversidad de cultura, costumbres, tradiciones, además es un gran exportador de materias primas que ayudan a potencializar el ingreso de devisas para el desarrollo de todos los ciudadanos que residen en el país.

\section{Referencias bibliográficas.}

Pérez , J., \& Gardey, A. (2012). concepto de migracion. Obtenido de https://definicion.de/migracion/

Aguirre, F. G. (1998). campesinos, de que hablamos. Obtenido de https://www.uv.mx/iihs/files/2012/11/Cuaderno5.pdf

Ana, V. (2018). libros de texto ministerio de educación. Obtenido de https://educacion.gob.ec/wp-content/uploads/downloads/2018/08/Sociales-texto9no-EGB.pdf

Cisneros, C., Preston, D., \& Ibarra, H. (1985). Población, migración y empleo en el Ecuador. Obtenido de http://biblio.flacsoandes.edu.ec/catalog/resGet.php?resId=47541

Cobo, J. F. (2004). migración campo-ciudad. Obtenido de https://www.puce.edu.ec/economia/docs/disertaciones/2004/2004_falconi_cobo_joh anna.pdf 
Cunti, H. (2007). Historia del Ecuador. Obtenido de https://www.educar.ec/noticias/hisecuador.pdf

Enrique, A. (2008). historia del ecuador. Obtenido de http://repositorio.uasb.edu.ec/bitstream/10644/836/1/AYALAE-CON0001-

RESUMEN.pdf;resumen

Mari., A. S. (2007). Caracteristicas de las zonas Urbanas. Obtenido de https://socialpcpi2.files.wordpress.com/2010/02/bloque-vi-lugares-distintos.pdf

norma, f. r., karl, m., \& kenra, f. (s.f.).

Pérez, J., \& Gardey, A. (2017). Definicion de Campesino. Obtenido de https://definicion.de/campesino/

Pérez, J., \& Gardey, A. (2017). Drfinicion de campesino. Obtenido de https://definicion.de/campesino/

Peréz, J., \& Merino, M. (2012). Definicion de zona rural. Obtenido de https://definicion.de/zona-urbana/

Perruchoud, R. (2006). Glosario sobre la migracion. Obtenido de https://publications.iom.int/system/files/pdf/iml_7_sp.pdf

Sanz , N., \& Valenzuela, J. M. (2016). Migracion y Cultura. Obtenido de http://unesdoc.unesco.org/images/0024/002477/247760s.pdf

Wolf., E. (1966). Para una definicion sociológica del campesino. Obtenido de https://helvia.uco.es/bitstream/handle/10396/5625/a001_01.pdf?sequence=1

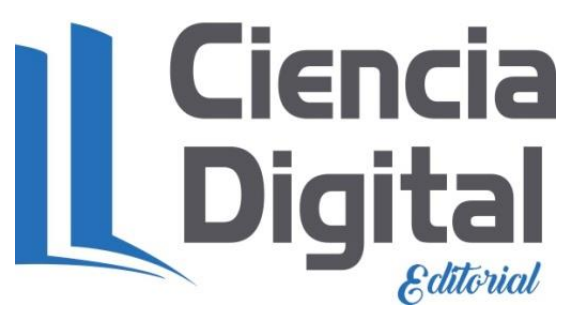


Para citar el artículo indexado.

Carrasco Ruano, Y. (2019). La migración interna de los campesinos hacia las zonas urbanas en el Ecuador. ConcienciaDigital, 2(3), 65-79.

https://doi.org/10.33262/concienciadigital.v2i3.932

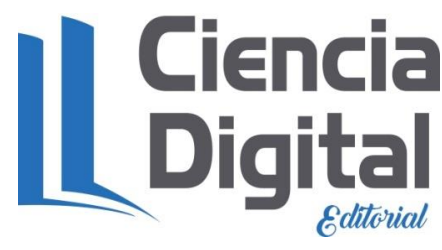

El artículo que se publica es de exclusiva responsabilidad de los autores y no necesariamente reflejan el pensamiento de la Revista Conciencia Digital.

El articulo queda en propiedad de la revista y, por tanto, su publicación parcial y/o total en otro medio tiene que ser autorizado por el director de la Revista Conciencia Digital.
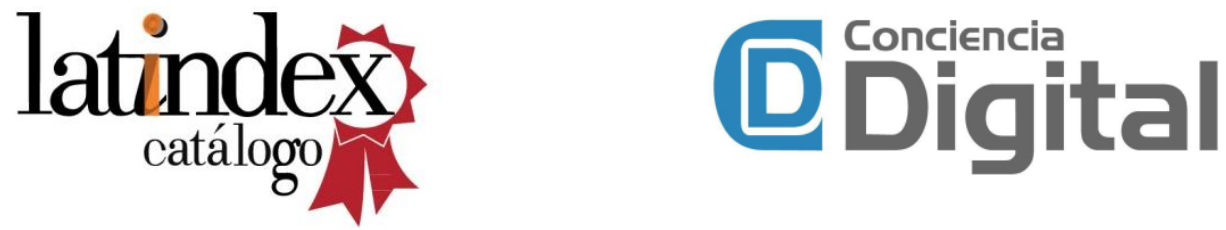